\title{
Asymmetric Loss in the Greenbook and the Survey of Professional Forecasters*
}

\author{
Yiyao Wang ${ }^{\dagger}$ and Tae-Hwy Lee \\ First version: March 2012 \\ Second version: November 2012 \\ This version: July 2013
}

\begin{abstract}
This paper examines forecast rationality of the Greenbook and the Survey of Professional Forecasters (SPF) under asymmetric loss functions, using the method proposed by Elliott, Komunjer and Timmermann (2005) with a rolling window strategy. Over rolling periods, the degree and direction of asymmetry in forecast loss function are time-varying. While rationality under symmetric loss is often rejected, forecast rationality under asymmetric loss is not rejected over nearly all rolling periods. Besides, real output growth is consistently under-predicted in 1990s and inflation rate is consistently over-predicted in 1980s and 1990s. Generally, inflation forecast, especially for long horizon, exhibits greater level of loss asymmetry in magnitude and frequency. The loss asymmetry of real output growth forecast is more pronounced when the last revised vintage data is used rather than real-time vintage is used. All of these results similarly hold in Greenbook and SPF. The results are also similar with different sets of instrumental variables for estimation of the asymmetric loss and for forecast rationality test.
\end{abstract}

Key Words: Greenbook, SPF, Asymmetric loss, Forecast rationality, Real output growth forecast, Inflation rate forecast, Real time data, Revised data.

JEL Classification: C53, E37

${ }^{*}$ The authors would like to thank the editor, Graham Elliott, and two anonymous referees for many helpful comments and suggestions.

${ }^{\dagger}$ Booth School of Business, the University of Chicago, Chicago, IL 60637. E-mail: yiyaowang@chicagobooth.edu

${ }^{\ddagger}$ Corresponding author. Department of Economics, University of California, Riverside, CA 92521, USA. E-mail: taelee@ucr.edu 


\section{Introduction}

Some forecasting research takes the forecast producer's perspective and deals with issues about constructing optimal forecast under a given loss function. Other research takes the forecast user's perspective and deals with issues about testing forecast rationality of a forecast producer. Since various forecasts for economic variables are made available to public, e.g., government forecasts such as Greenbook, private-sector forecasts such as Survey of Professional Forecasters (SPF), and forecasts of international organizations such as IMF and OECD, the latter view from the forecast user's perspective, to test forecast rationality, becomes an important research topic.

Forecast rationality has been often tested under a given symmetric loss function. For example, under symmetric squared error loss, the rational forecast is unbiased, the single-period horizon forecast errors are serially uncorrelated, and the unconditional variance of the forecast error is a non-decreasing function of forecast horizon (cf. Granger and Newbold 1986, Diebold and Lopez 1996, Patton and Timmermann 2005). These properties make it convenient to test for forecast rationality. Romer and Romer (2000) provide support for the unbiasedness of Greenbook inflation forecast by applying the Mincer-Zarnowitz (1969) test, while in Capistran (2008), rationality of Greenbook inflation forecast is rejected in sub-periods. Rossi (2012) further discusses the timevarying nature of the unbiasedness result. Patton and Timmermann (2012) develop a new testing strategy based on properties of optimal multi-horizon forecasts under the symmetric squared error loss and reject rationality of Greenbook multi-horizon forecasts for quarter-over-quarter changes in GDP, GDP deflator and CPI. Croushore (2010) rejects forecast rationality of SPF inflation forecast under the symmetric squared error loss.

Forecast rationality can be tested under a specific asymmetric loss function. If a forecast can only be rationalized with assuming asymmetric loss, the rejection of forecast rationality under symmetric loss may probably be caused by false assumption of the symmetric loss rather than lack of forecast rationality. One way to deal with asymmetric loss is to develop new properties to test for forecast rationality, cf. Patton and Timmermann (2007b). Another way is to assume a particular asymmetric loss function. For example, Patton and Timmermann (2007a) use the Linex loss function of Varian (1975) with a regime switching model to develop properties to test for rationality.

Forecast rationality can also be tested without assuming a particular loss function. Elliott, Komunjer and Timmermann (2005, EKT henceforth) propose a method to estimate the loss function parameter from a large class of asymmetric loss functions, and develop a test for rationality under 
the estimated loss. This novel method sets few restrictions on the data generating process and estimates the asymmetric loss parameter using public information only. EKT discover that, allowing asymmetric loss, forecast rationality is rarely rejected in IMF and OECD forecasts of budget deficits for G7 countries. Following EKT, some extensions and consequent empirical work have been added. Patton and Timmermann (2007b) introduce a more flexible asymmetric loss function which depends not solely on forecast error but also on realized target value. Under such kind of loss functions, Greenbook real output forecast can be perfectly rationalized. Elliott, Komunjer and Timmermann (2008) apply the EKT method to SPF forecasts of nominal and real output growth and find that "only a modest degree of asymmetry is required for the survey expectations to be consistent with rationality". Komunjer and Owyang (2012) extend the EKT framework with a multivariate nonseparable asymmetric loss function to test forecast rationality of multivariate forecasts jointly.

Moreover, several papers have provided evidence that the degree of asymmetry estimated by EKT may not be constant over time. Capistran (2008) separates the full data period of Greenbook inflation forecast into 2 sub-periods, pre-Volcker and since-Volcker (Paul Volcker was the FRB chairman from 1979 to 1987), and conducts the EKT method separately for each period. He finds a significant difference in direction of asymmetry between the two periods and suggests that "the cost of having inflation above an implicit time-varying target was larger than the cost of having inflation below it for the since-Volcker period, and that the opposite was true for the pre-Volcker era." Patton and Timmermann (2007b) show that the level of loss asymmetry in Greenbook real output growth forecast in recession time is much higher than that in a high GDP period.

Following the above literature, this paper further studies the possible time-varying nature of loss function asymmetry and forecast rationality of Greenbook and SPF by applying the EKT method. First, we adopt a rolling window strategy and find that the potential level of loss asymmetry is time-varying. In rolling periods, rationality under symmetric loss is often rejected, but rationality under asymmetric loss is rarely rejected. This confirms EKT (2005) and Capistran (2008) that the asymmetry in loss function reflects forecasters' cautious risk attitude rather than forecast irrationality. Second, both real output growth forecast and inflation rate forecast are included in our analysis. We find that real output growth forecasts in 1990s are produced with a loss function that punishes over-predicting more than under-predicting, which leads to consistent underprediction, while the inflation forecasts in 1980s and 1990s are produced with an asymmetric loss in the opposite direction. Generally, inflation forecasts, especially for long horizon, embrace a higher 
level of asymmetry in magnitude and frequency. Third, we use different vintages for the realized data and discover that loss asymmetry of real output growth forecast is more pronounced when the last revised vintage data is used rather than when real-time vintage is used. Fourth, both Greenbook and SPF forecasts are included in our analysis of forecast rationality under asymmetric loss. The results suggest that there is clear similarity in the time-varying loss asymmetry pattern between the two forecasts, which may be valuable information because the SPF is available 5-year ahead before the publication of Greenbook. Fifth, these results are also similar with different sets of instrumental variables for estimation of the asymmetric loss and for test of forecast rationality.

The rest of the paper is organized as follows. Sections 2 describes data of Greenbook and SPF for forecast values as well as revised and real-time vintages for realized values. Section 3 provides a brief review of the EKT (2005) method. Section 4 presents a list of main empirical findings. Section 5 concludes.

\section{Data}

Let $f_{t+h}$ be the $h$-step-ahead forecast of $y_{t+h}$. We obtain the forecast value $f_{t+h}$ from the Greenbook or the SPF. The frequency of forecasts and realized values is quarterly at the source. The realized values $y_{t+h}$ of real output growth or inflation rate are obtained from the real-time data vintages or from the most recently revised data vintage.

Greenbook forecast is produced by the Board of Governors of the Federal Reserve Board before each meeting of the Federal Open Market Committee, starting from the fourth quarter of 1965. As in Sims (2002), the forecast project is broken down into forecasts of several sub-variables for different Fed experts to model and forecast. Sub-variable forecasts are combined together by a primary macroeconomic model named FRB/US to generate original Greenbook forecasts of several economic variables for further judgmental adjustment and feedback. Since the Greenbook may have a substantial influence on policy making, it is made available to public after a lag of five years. Although the FOMC meeting is more frequent than four times a year with a varying time schedule, we use the quarterly Greenbook forecasts, which is compatible with the SPF frequency. In our analysis, forecasts for both real output growth in GNP/GDP index and inflation rate in GNP/GDP price index are included. ${ }^{1}$ Greenbook covers a variety of horizons up to 8 or 9 quarters, yet we only consider horizons of $h=1,4$, representative for short and long horizon forecasts. For each horizon, the data period is different: one-quarter-ahead forecast is available from 1968Q3 to

\footnotetext{
${ }^{1}$ Both variables are annualized percentage value of quarter-over-quarter growth rate. The forecasts were for GNP from 1965 to 1991 and for GDP from 1992 and on.
} 
2005Q4, and four-quarter-ahead is available from 1974Q2 to 2005Q4. Largest data period for each horizon is used in order to take advantages of all information.

In addition to the Greenbook forecast, we also use real output growth forecast and inflation rate forecast with horizons $h=1,4$ from the Survey of Professional Forecasters (SPF), a well-known survey forecast currently organized by the Federal Reserve Bank of Philadelphia. The SPF is a set of many forecasts, mostly made by professional forecasters from business companies or the Wall Street. In SPF, one-quarter-ahead forecast is available from 1968Q4 to 2012Q1 at the time of our writing this paper, and four-quarter-ahead forecast is available from 1974Q4 to 2012Q1. ${ }^{2}$ Notably, the forecast for real output growth actually started from 1981Q3, before which the forecast values were computed by the forecast of nominal GNP and GNP price deflator index. Different from the Greenbook, recent SPF forecasts are available without lag, which might provide a valuable source of forecast in the absence of Greenbook forecast. Because SPF consists of forecasts from many professional forecasters, there is a certain degree of dispersion between different individual forecasters, researched by Capistran and Timmermann (2009) and Patton and Timmermann (2010). In this paper, the median response of the forecast survey is used as a consensus of the SPF forecasts.

Both "real-time data" and "revised data" are used in this paper as realized value $y_{t+h}$. Realtime data is name for the second revision of statistical data, systematically proposed and discussed by Croushore and Stark (2001) and provided in the Real-Time Data Research Center of Federal Reserve Bank of Philadelphia. Data of the current quarter's growth rate are computed in the following quarter's vintage. The second revision data are comparatively complete with less statistical error compared to initial revision data, and are also closer to what the forecasters are forecasting, compared to later revision, because "this series does not include the rebenchmarking and definitional changes that occur in the annual and quinquennial revisions" (Romer and Romer 2000). On the other side, "revised data" are computed with the most recent vintage (2011Q4 vintage in this paper), which should well represent the economic status of past periods on the standpoint of today. Indexes of real GNP/GDP and GNP/GDP price are made available in the website of Federal Reserve Bank of Philadelphia. Applying the transformation of $y_{t}=400 \times \ln \left(x_{t} / x_{t-1}\right)$ with $x$ being either the real GNP/GDP or GNP/GDP price index, we obtain data of real output growth and inflation rate, both real-time and revised, compatible in format with forecast values.

\footnotetext{
${ }^{2}$ However, the realized value is only available before and including 2011Q3, the last few forecasts of SPF are not used in our empirical study - the data periods used in this paper should be: 1968Q4 to 2011Q2 (SPF 1-step-ahead) and 1974Q4 to 2010Q3 (SPF 4-step-ahead).
} 


\section{The EKT Method}

The EKT method is based on linear GMM framework. Here we briefly review the method. Let $f_{t+h}=\theta^{\prime} W_{t}$ be the $h$-step-ahead linear forecast of $y_{t+h}$ conditional on the information set $\mathcal{F}_{t}$ at time $t$, where $\theta$ is an unknown $k$-vector of parameters and $W_{t}$ is a $k$-vector of variables that are $\mathcal{F}_{t}$-measurable. A generalization to nonlinear forecast $f_{t+h}=\theta\left(W_{t}\right)$ and nonlinear GMM is straightforward. EKT use a flexible class of loss function

$$
L(\alpha, p, \theta)=\left[\alpha+(1-2 \alpha) \cdot 1\left(\varepsilon_{t+h}<0\right)\right] \cdot\left|\varepsilon_{t+h}\right|^{p}
$$

where $\alpha \in(0,1)$ and $p$ is a positive integer. We present the results with $p=2.1(\cdot)$ is the indicator function which equals to 1 when its argument is true and 0 otherwise, and $\varepsilon_{t+h}=y_{t+h}-f_{t+h}$ is the forecast error. The parameter $\alpha$ depicts the level of loss asymmetry. When $\alpha<0.5$, forecasters tend to punish over-prediction more and create a bias towards under-prediction. When $\alpha>0.5$, forecasters tend to punish under-prediction more and create a bias toward over-prediction. For given $\left(\alpha_{0}, p_{0}\right)$, the forecast $f_{t+h}^{*}=\theta^{* \prime} W_{t}$ is rational if $\theta^{*}=\arg \min _{\theta \in \Theta} E\left\{L\left(\alpha_{0}, p_{0}, \theta\right)\right\}$ solves the following first order condition (FOC)

$$
E\left(W_{t} \cdot\left[1\left(y_{t+h}-f_{t+h}^{*}<0\right)-\alpha_{0}\right] \cdot\left|y_{t+h}-f_{t+h}^{*}\right|^{p_{0}-1}\right)=0 .
$$

Given the forecast $\hat{f}_{t+h}=\hat{\theta}_{t}^{\prime} W_{t}$ provided from its producer (such as FRB or SPF), the forecast user wishes to estimate $\alpha$ of the producer's loss function with a given value of $p_{0}$. In order to back out $\alpha$, the FOC should hold if and only if $\alpha=\alpha_{0}$. EKT prove that $\theta^{*}\left(\alpha_{0}\right)$ is a continuous differentiable one-to-one mapping from $(0,1)$ to $\Theta$. This indicates that a different level of loss asymmetry, $\alpha$, yields a different forecast and a different forecast will reveal a different level of loss asymmetry. $W_{t}$ is the information set that the forecaster knows at time $t . k=\operatorname{dim}\left(W_{t}\right)$ is typically very large, and not all of information in $W_{t}$ is accessible to the users.

Instruments for $W_{t}$ : To estimate $\alpha_{0}$, we only need $m=\operatorname{dim}\left(V_{t}\right)(\geq 1)$ instruments $V_{t}$, satisfying the condition

$$
A\left(\alpha_{0}\right) \equiv E\left(V_{t} \cdot\left[1\left(y_{t+h}-f_{t+h}^{*}<0\right)-\alpha_{0}\right] \cdot\left|y_{t+h}-f_{t+h}^{*}\right|^{p_{0}-1}\right)=0
$$

We have considered several sets of instrumental variables $V_{t}$ including the following sets: $V_{t}=1$

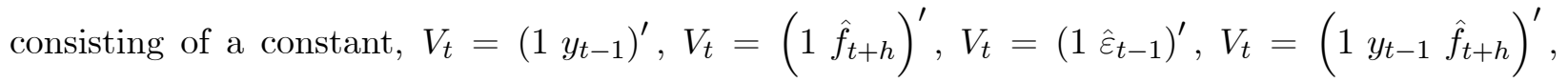
$V_{t}=\left(\begin{array}{lll}1 & y_{t-1} & \hat{\varepsilon}_{t-1}\end{array}\right)^{\prime}$, and $V_{t}=\left(\begin{array}{lll}1 & y_{t-1} & y_{t-1}^{2}\end{array}\right)^{\prime}$. As $V_{t}=W_{t}$ is optimal, $V_{t}=f_{t+h}^{*}=\theta^{* \prime} W_{t}$ would be a good instrument if $\theta^{*}$ were known. In reality $\theta^{*}$ and $f_{t+h}^{*}$ are not known, yet it may be possible 
that $V_{t}=\hat{f}_{t+h}=\hat{\theta}_{t}^{\prime} W_{t}$ could be a good instrument as $\hat{\theta}_{t}$ is measurable with respect to $W_{t}$ (and so is $\left.\hat{f}_{t+h}\right)$. Hence, $V_{t}=\left(1 \hat{f}_{t+h}\right)^{\prime}$ and $V_{t}=\left(1 y_{t-1} \hat{f}_{t+h}\right)^{\prime}$ are valid instruments satisfying the moment condition (3). If a rational forecast $f_{t+h}\left(\theta, W_{t}\right)$ is nonlinear in $W_{t}$, the above FOC can be modified to replace $W_{t}$ with the gradient of $f_{t+h}\left(\theta, W_{t}\right)$ with respect to $\theta$, evaluated at $\left(\theta^{*}, W_{t}\right)$, as noted in $\operatorname{EKT~(p.~1110).~For~example,~if~} f_{t+h}\left(\theta, W_{t}\right)=\theta_{0}+\theta_{1} y_{t-1}+\theta_{2} y_{t-1}^{2}$ then the instrument can be taken as $V_{t}=\left(\begin{array}{lll}1 & y_{t-1} & y_{t-1}^{2}\end{array}\right)^{\prime}$. Overall, the similar results are obtained with these different choices of the instruments. The instruments with $\hat{f}_{t+h}$ yield somewhat unstable estimates of $\alpha$ with some large changes in $\hat{\alpha}_{T, \tau}$ over the rolling windows, especially when $h=4$, which may be due to the small sample size $T=40$ for the estimation windows. We present the empirical results with $V_{t}=\left(\begin{array}{ll}1 & y_{t-1}\end{array}\right)^{\prime}$ in the next section, with brief remarks on the results from using the other sets of instrumental variables (which are available in a supplemental appendix at the authors' websites).

Backing out the asymmetric parameter: Let $B=E\left(V_{t} \cdot\left|y_{t+h}-f_{t+h}^{*}\right|^{p_{0}-1}\right)$ and $C=$ $E\left(V_{t} \cdot 1\left(y_{t+h}-f_{t+h}^{*}<0\right) \cdot\left|y_{t+h}-f_{t+h}^{*}\right|^{p_{0}-1}\right)$. Write $A(\alpha) \equiv C-\alpha B$. The orthogonality condition (3) can be solved from minimizing $Q(\alpha)=A(\alpha)^{\prime} S^{-1} A(\alpha)$ which yields $\alpha_{0}=\left(B^{\prime} S^{-1} C\right) /\left(B^{\prime} S^{-1} B\right)$. This can be estimated by $\hat{\alpha}_{T, \tau}=\left(\hat{B}_{T, \tau}^{\prime} \hat{S}_{T, \tau}^{-1} \hat{C}_{T, \tau}\right) /\left(\hat{B}_{T, \tau}^{\prime} \hat{S}_{T, \tau}^{-1} \hat{B}_{T, \tau}\right)$ where $\hat{B}_{T, \tau}=\frac{1}{T} \sum_{t=\tau}^{T+\tau-1} V_{t}$. $\left|y_{t+h}-\hat{f}_{t+h}\right|^{p_{0}-1}, \hat{C}_{T, \tau}=\frac{1}{T} \sum_{t=\tau}^{T+\tau-1} V_{t} \cdot 1\left(y_{t+h}-\hat{f}_{t+h}<0\right) \cdot\left|y_{t+h}-\hat{f}_{t+h}\right|^{p_{0}-1}$, and $\hat{S}_{T, \tau}$ is a consistent estimate of $S$. The estimate $\hat{\alpha}_{T, \tau}$ depends on the estimation of $S$, which in turn depends on $\alpha_{0}$, so we iterate estimation of $\alpha$ and the Newey-West (1987) estimator of $S$. EKT establishes consistency and asymptotic normality: $T^{\frac{1}{2}}\left(\hat{\alpha}_{T, \tau}-\alpha_{0}\right) \stackrel{d}{\rightarrow} N\left(0,\left(B^{\prime} S^{-1} B\right)^{-1}\right)$.

Rolling windows: We apply the rolling window strategy to examine possible time varying behavior of the asymmetric loss parameter and to analyze its asymmetry preference in different periods of time. Let $\tau$ denote the beginning of a rolling estimation sample and $T$ denote the size of the rolling estimation sample, and the index $t$ is used to denote the time point when an $h$-stepahead forecast $\hat{f}_{t+h}$ is made. Let $n+h$ be the total number of periods available. We use the first rolling sample $\left\{V_{t}, y_{t+h}, \hat{f}_{t+h}\right\}_{t=2}^{t=T+1}$ to compute $\hat{\alpha}_{T, 1}$, the $\tau$ th rolling sample $\left\{V_{t}, y_{t+h}, \hat{f}_{t+h}\right\}_{t=\tau+1}^{t=T+\tau}$ to compute $\hat{\alpha}_{T, \tau}$, and the last rolling sample $\left\{V_{t}, y_{t+h}, \hat{f}_{t+h}\right\}_{t=n-T+1}^{t=n}$ to compute $\hat{\alpha}_{T, n-T}$. Hence, we obtain the total $n-T$ number of the asymmetric loss parameter estimates $\hat{\alpha}_{T, \tau}$ where the first index $T$ denotes the size of the rolling window and the second index $\tau=1, \ldots, n-T$ denotes the time when the rolling window begins. In presenting $\left\{\hat{\alpha}_{T, \tau}\right\}$ in Section 4, we use figures whose horizontal axis be $\tau=1, \ldots, n-T$, with a fixed $T=40$ (40 quarters in a 10 year window) and $n$ being determined by the maximum length of the forecasts available in Greenbook and SPF. In applying the rolling window scheme, we move the period one quarter at a time to compute the 
corresponding estimate of the asymmetric parameter until we reach the end of the data period.

Rationality test under asymmetric loss: We construct the J-statistic for rationality test under the asymmetric loss,

$$
J_{T, \tau}\left(\hat{\alpha}_{T, \tau}\right)=T \times \hat{Q}_{T, \tau}\left(\hat{\alpha}_{T, \tau}\right)=T \times \hat{A}_{T, \tau}\left(\hat{\alpha}_{T, \tau}\right)^{\prime} \hat{S}_{T, \tau}^{-1}\left(\hat{\alpha}_{T, \tau}\right) \hat{A}_{T, \tau}\left(\hat{\alpha}_{T, \tau}\right),
$$

where $\hat{A}_{T, \tau}\left(\hat{\alpha}_{T, \tau}\right)=\hat{C}_{T, \tau}-\hat{\alpha}_{T, \tau} \hat{B}_{T, \tau}$. This J-test for over-identification checks whether the orthogonality condition (3) holds for $\alpha=\hat{\alpha}_{T, \tau}$, that is to check whether forecast rationality holds for $\alpha=\hat{\alpha}_{T, \tau}$. If the number of orthogonal conditions (the number of instruments) is larger than one, the $J_{T, \tau}\left(\hat{\alpha}_{T, \tau}\right)$ statistic follows the asymptotic chi-square distribution with $(m-1)$ degrees of freedom, $\chi_{m-1}^{2}$. A large value of the $J_{T, \tau}\left(\hat{\alpha}_{T, \tau}\right)$ statistic would indicate the rejection of forecast rationality condition (3) when $\alpha=\hat{\alpha}_{T, \tau}$.

Rationality test under symmetry: Conditioning that the $J_{T, \tau}\left(\hat{\alpha}_{T, \tau}\right)$ statistic has not rejected the orthogonality condition (3), the $J_{T, \tau}(0.5)$ statistic with $\alpha=0.5$ can be used to test for rationality under loss symmetry. The rejection of this test would point to the rejection of the loss symmetry if the rationality has not been rejected by $J_{T, \tau}\left(\hat{\alpha}_{T, \tau}\right)$. However, the conditional distribution of the restricted statistic $J_{T, \tau}(0.5)$ conditional on $J_{T, \tau}\left(\hat{\alpha}_{T, \tau}\right)$ is hard to obtain. Hence, the statistic $J_{T, \tau}(0.5)$ is taken as a joint test statistic for forecast rationality and loss symmetry. In this case, $J_{T, \tau}(0.5)$ follows asymptotically the chi-square distribution $\chi_{m}^{2}$ (with degrees of freedom equal to $m$ as no parameter has been estimated). In the next section however, we will loosely interpret $J_{T, \tau}(0.5)$ as a test for loss symmetry. The test for loss symmetry may also be conducted by computing the $95 \%$ confidence interval of $\alpha_{0}$ using the asymptotic normality result of $\hat{\alpha}_{T, \tau}$.

It may be noted that allowing for a time-varying asymmetry parameter does not necessarily imply that forecast rationality will be satisfied. When in fact the symmetry is not true $\left(\alpha_{0} \neq 0.5\right)$, it is true that the power of the rationality test $J_{T, \tau}(0.5)$ with assuming symmetry would be higher, rejecting forecast rationality more often than the forecast rationality test $J_{T, \tau}\left(\hat{\alpha}_{T, \tau}\right)$ with allowing time-varying asymmetry. However, $J_{T, \tau}\left(\hat{\alpha}_{T, \tau}\right)$ will still have power against departures from forecast rationality as it is designed for. On the other hand, when in fact the symmetry is true $\left(\alpha_{0}=0.5\right)$, the power of the rationality test $J_{T, \tau}(0.5)$ with symmetry being assumed could be lower, rejecting forecast rationality less often than the forecast rationality test $J_{T, \tau}\left(\hat{\alpha}_{T, \tau}\right)$ with allowing time-varying asymmetry. ${ }^{3}$

\footnotetext{
${ }^{3} J_{T, \tau}(0.5)$ can be computed using $\hat{\alpha}_{T, \tau}=0.5$ in eq (4). However, we use $\hat{S}_{T, \tau}\left(\hat{\alpha}_{T, \tau}\right)$ instead of $\hat{S}_{T, \tau}(0.5)$ which can improve the finite sample power, as noted in EKT (p. 1114).
} 


\section{Empirical Analysis}

We apply the EKT method to full data period as well as rolling windows of size $T=40$. Three statistics are computed: the asymmetric loss parameter estimate $\hat{\alpha}_{T, \tau}$ with standard error, the p-value of the statistic $J_{T, \tau}\left(\hat{\alpha}_{T, \tau}\right)$ for forecast rationality under asymmetric loss, and the p-value of the joint test statistic $J_{T, \tau}(0.5)$ for forecast rationality and loss symmetry. The results for full data period are reported in Table 1, and the results for rolling windows are reported in Figures 1-4.

Table 1 about here

Table 1 presents results for the full data period. The estimates $\hat{\alpha}_{T, \tau}$ are followed by their standard errors $s e\left(\hat{\alpha}_{T, \tau}\right)$ in brackets, and $J_{T, \tau}(0.5)$ and $J_{T, \tau}\left(\hat{\alpha}_{T, \tau}\right)$ are followed by p-values reported in brackets. For the forecast value $\hat{f}_{t+h}$, both the Greenbook and the SPF forecasts (of real output growth and inflation rate) are included. For the realized values of $y_{t+h}$, both real-time data and revised data are used. Results with both 1-step-ahead (one-quarter-ahead) forecast and 4-stepahead (one-year-ahead) forecast are presented. Most estimates of the asymmetric loss parameter are near 0.5. P-values of $J_{T, \tau}(0.5)$ which tests rationality under symmetry are larger than 0.05, except for those of $h=4 \mathrm{SPF}$ inflation forecast. This indicates that for most cases with full data period, the asymmetry in forecast loss function of Greenbook and SPF is not statistically significant.

Figures 1, 2, 3, 4 about here

Figure 1-4 present results for the rolling sub-periods. Figures 1 and 2 present results for each rolling window indexed by $\tau$ for real output growth forecasts with $h=1$ and $h=4$, respectively, and Figures 3 and 4 do the same for inflation rate forecasts. Each figure has a $4 \times 2$ array of 8 panels of time series graphs over $\tau$. Column 1, (a), (c), (e), (g) in the left panels, reports "Estimates" $\hat{\alpha}_{T, \tau}$ with the $95 \%$ asymptotic confidence intervals at each $\tau$. Column 2 , (b), (d), (f), (h) in the right panels, reports the p-values of two "Tests", $J_{T, \tau}\left(\hat{\alpha}_{T, \tau}\right)$ and $J_{T, \tau}(0.5)$. The p-values of the statistic $J_{T, \tau}\left(\hat{\alpha}_{T, \tau}\right)$ are in lighter (red) color and the p-values of the statistic $J_{T, \tau}(0.5)$ are in darker (black) color. Each figure has four rows with different pairs of forecast value and realized value. ${ }^{4}$ In all figures, the time stamp in the horizontal axis, denoted by $\tau$, is the beginning of the 10 year rolling windows as discussed in the previous section.

\footnotetext{
${ }^{4}$ Row 1 uses the forecasts $\hat{f}_{t+h}$ from Greenbook and the realized data $y_{t+h}$ from real-time vintages. Row 2 uses the forecasts $\hat{f}_{t+h}$ from Greenbook and the realized data $y_{t+h}$ from the last revised data vintage. Row 3 uses the forecasts $\hat{f}_{t+h}$ from SPF and the realized data $y_{t+h}$ from real-time vintages. Row 4 uses the forecasts $\hat{f}_{t+h}$ from SPF and the realized data $y_{t+h}$ from revised data.
} 
The asymmetric loss parameter estimates $\hat{\alpha}_{T, \tau}$ are apparently time-varying in degree and direction, as shown in Column 1 of the four figures. Different from results with full data period in Table 1, forecast rationality under symmetry is often rejected in rolling periods, given the fact that the $95 \%$ confidence intervals often do not include $\alpha=0.5$ (dashed line) and that p-values of $J_{T, \tau}(0.5)$ in Column 2 are often smaller than 0.05 (dashed line), especially in inflation forecast. While the values of $\hat{\alpha}_{T, \tau}$ are mostly below 0.5 for real output growth forecast and mostly above 0.5 for inflation rate forecasts, occasional crossings are also observed.

In Column 2 of all four figures, although the joint hypothesis of symmetry and rationality for many rolling periods is rejected due to small p-values of $J_{T, \tau}(0.5)$, p-values of $J_{T, \tau}\left(\hat{\alpha}_{T, \tau}\right)$ for forecast rationality under asymmetric loss rarely go below 0.05 and are much larger than their counterparts, $J_{T, \tau}(0.5)$. Rejection of forecast rationality occurs only in a very few rolling windows. This implies that in most rolling periods, forecast rationality under asymmetric loss is not rejected for both real output growth forecast and inflation rate forecast, for both one quarter horizon and one year horizon, and for both real-time data and revised data. This finding confirms that the rejection of the joint test of symmetry and rationality is likely due to the rejection of the symmetry rather than the rejection of forecast rationality.

There is a substantial difference in the pattern of time-varying asymmetry between real output growth forecast and inflation rate forecast. For real output growth in Figures 1-2, the level of loss asymmetry is generally mild, except those periods around $\tau=1990 Q 1$ when the estimates of $\hat{\alpha}_{T, \tau}$ are significantly below 0.5 in Column 1 . It suggests that an asymmetric loss function that punishes over-predicting more than under-predicting was used in 1990s. For inflation rate, there is much greater asymmetry in terms of magnitude and frequency. In Figures 3-4, forecast rationality under symmetric loss is consistently rejected over a wide range of periods including 1980s and 1990s. The estimates $\hat{\alpha}_{T, \tau}$ in Column 1 are significantly above 0.5. This indicates that the forecaster consistently punishes under-predicting much more than over-predicting inflation rate during 1980s and 1990s. Notably, in inflation forecast, long-horizon inflation forecast exhibits a greater level of loss asymmetry than short-horizon inflation forecast. We have computed the same statistics (not reported for space) for inflation nowcast $(h=0)$ of Greenbook and SPF and find that the level of loss asymmetry is much milder in inflation nowcast, compared to inflation forecast of longer horizons.

A surprising similarity in the asymmetric loss preference between Greenbook and SPF in most rolling periods is observed from the graphs. For all pairs of Greenbook and SPF forecasts, the 
estimates $\hat{\alpha}_{T, \tau}$ in Column 1 generally share the same pattern. For example, compare Panel (a) and (e) of Figure 4. Both the estimates rise from below 0.5 to above 0.5 , become stably high above 0.5 at nearly the same time $\tau$ and drop to below 0.5 at nearly the same time $\tau$.

We summarize our results as follows: (1) Over rolling periods, the degree and direction of the asymmetry in forecast loss function are time-varying, and forecast rationality under symmetry is often rejected. (2) Forecast rationality under asymmetric loss is not rejected over nearly all rolling windows for both real output growth and inflation rate. (3) Real output growth is consistently under-predicted in 1990s, while inflation rate is consistently over-predicted in 1980s and 1990s. (4) Inflation forecast, especially that with long horizon, exhibits a greater level of loss asymmetry in both magnitude and frequency than output growth forecast. (5) The above results are similar between Greenbook and SPF.

These results are obtained similarly from using the other sets of instrumental variables as discussed in the previous section. Although we do not report them here for space reasons (available in the supplemental appendix), we make some remarks from using the different instruments. In all sets of instruments we considered, the standard errors of $\hat{\alpha}_{T, \tau}$ are typically small but they become sometimes smaller using $V_{t}=\left(1 \hat{f}_{t+h}\right)^{\prime}$ or $V_{t}=\left(1 \hat{\varepsilon}_{t-1}\right)^{\prime}$. The power of the forecast rationality test also becomes slightly higher than using $V_{t}=\left(\begin{array}{ll}1 & y_{t-1}\end{array}\right)^{\prime}$. Meanwhile, the estimates $\hat{\alpha}_{T, \tau}$ make more apparent abrupt changes in adjacent rolling periods when the instrument $V_{t}=\left(1 \hat{f}_{t+h}\right)^{\prime}$ is used especially in inflation forecasts. These abrupt changes in $\hat{\alpha}_{T, \tau}$ may be reflection of genuine timevarying nature of the asymmetry, can be due to quality of the instruments, and/or the small rolling window size $T=40$. Instruments with three elements also give similar results in the estimates of $\hat{\alpha}_{T, \tau}$ and in the test p-values. For example, the results from using $V_{t}=\left(\begin{array}{lll}1 & y_{t-1} \hat{f}_{t+h}\end{array}\right)^{\prime}$ are similar to those from using $V_{t}=\left(1 \hat{f}_{t+h}\right)^{\prime}$ or $V_{t}=\left(\begin{array}{lll}1 & y_{t-1}\end{array}\right)^{\prime}$. Adding $y_{t-1}^{2}$ we consider $V_{t}=\left(\begin{array}{lll}1 & y_{t-1} & y_{t-1}^{2}\end{array}\right)^{\prime}$, which gives slight edge in power of the forecast rationality test and smaller standard errors.

In addition, robustness of our results is checked in several dimensions. Our results are robust to the choice of $p$. The results with $p=1$ are similar to those with $p=2$, but with a milder level of loss asymmetry. Our results are also robust to the choice of $T$, the size of rolling windows. The rolling window of 40 quarters may be a value not too long to erase the difference between different time periods and also not too short to undermine the power of tests and the asymptotic results. With a small rolling window like $T=20$, the power of the rationality test might be severely undermined, while with large rolling window like $T=80$, the potential time variation in $\hat{\alpha}_{T, \tau}$ can be smoothed out. The results in the figures presented are with $T=40$ (quarters). The results with $T=20,80$ 
are similar to those presented and not reported for space.

Different vintages are applied to compute the realized value of real output growth and inflation rate. While our main results are qualitatively robust to the choice of data vintages, a major difference is observed with realized values of different vintages especially in real output growth forecast. In both Table 1 and four figures, the estimates of the loss function parameter for real output growth forecast are generally smaller with revised realized value than with real-time realized value for the forecast target variable. This difference can be explained by a upward trend of data revision after the second revision: the average real output growth from 1968Q4 to 2005Q4 in revised data exceeds that in real-time data by $0.52 \%$ in absolute value, while the difference is only $0.16 \%$ with inflation forecast. The upward trend of data revision in real output growth is large enough to have a significant effect on tests. Because the realized values $(y)$ in revised data are generally larger than those in real-time data, the forecast error $(\varepsilon=y-f)$ tends to be larger with revised data, so there are more under-predictions and less over-predictions with revised data, which in general amounts to smaller estimated values of $\alpha$ in rolling periods. The real-time data may be more appropriate to use in actual forecasting practice as "the data (in latest vintage) they are using have been revised over time and differ significantly from the data used by forecasters in real time" (Croushore and Stark 2001), therefore, using the revised data in realized real output growth would give estimates stronger level of asymmetry in forecast producer's loss function than what actually is. Specifically, the difference is most pronounced in Figure 1, the one-step-ahead real output growth forecast of both Greenbook and SPF, where we can see big differences depending on which version of the data is used.

\section{Conclusions}

This paper examines the asymmetry in forecast loss function of Greenbook and SPF over rolling periods. We find the degree and direction of the asymmetry in forecast loss function of Greenbook and SPF time-varying over rolling samples. It implies that using the full sample (as in Table 1) or assuming a constant asymmetry level may be misleading. In Capistran (2008), a huge difference is found in loss function parameter of Greenbook inflation forecast between two sub-periods. This paper examines the time-variation of the loss function parameter of forecast producers using rolling windows, which offers more pictures of the asymmetry in direction, magnitude and frequency over time. This paper also confirms that Greenbook and SPF forecasts of real output growth and inflation rate are rationalizable if asymmetry is permitted in the loss function of these forecast 
agents. This finding is in line with a finding of Elliott, Komunjer and Timmermann (2008) that asymmetry in loss function is necessary to obtain forecast rationality for SPF forecasts of nominal and real output growth. These time-varying asymmetry results are similar with different sets of instrumental variables for the estimation of the asymmetric loss and for the test of forecast rationality.

One interesting result is the asymmetry of under-prediction in real output growth during 1990s and over-prediction in inflation rate during 1980s and 1990s. For Greenbook, this long period of significant over-prediction of inflation rate coincides with a strict monetary policy during 1980s and 1990s and with consequent low inflation. Romer and Romer (2004) argue that "the welltempered monetary policies of the 1950s and of the 1980s and 1990s stemmed from a conviction that inflation has high costs and few benefits, together with realistic views about the sustainable level of unemployment and the determinants of inflation." According to this, Greenbook's overprediction of inflation, reflected in its asymmetric loss, is preemptive, leading to a policy that is intended to lower inflation.

Another interesting result is that, when different vintages are used for the realized value of real output growth, the level of loss asymmetry is more pronounced for real output growth forecast when the last vintage (revised) data are used rather than when the real-time vintage data are used. Without using the real-time data in evaluating forecasts of real output growth, the loss asymmetry can be significantly exaggerated.

Finally, it is useful to note the similarity in loss preference between Greenbook and SPF, which may be a consequence from SPF's keeping up with Greenbook in terms of loss preference hoping to benefit the private sectors (producers of SPF forecasts) from future monetary policy of FRB (the producer of the Greenbook). Due to this similarity, the information contained in SPF may be valuable since Greenbook forecasts are published with five year delay. It remains to be seen whether the recent directional change in the SPF loss preference can foretell the changes in the loss preference of Greenbook. 


\section{References}

Capistran, C. (2008), "Bias in Federal Reserve Inflation Forecasts: Is the Federal Reserve Irrational or Just Cautious?", Journal of Monetary Economics 55(8): 1415-1427.

Capistran, C. and Timmermann, A. (2009), "Disagreement and Biases in Inflation Expectations", Journal of Money, Credit and Banking 41(2-3): 365-396.

Croushore, D. (2010), "An Evaluation of Inflation Forecasts from Surveys Using Real-Time Data", B.E. Journal of Macroeconomics, Contributions 10, Article 10.

Croushore, D. and Stark, T. (2001), "A Real-Time Data Set for Macroeconomists", Journal of Econometrics 105: 111-130.

Diebold, F. X. and Lopez, J. (1996), "Forecast Evaluation and Combination", in Handbook of Statistics, eds. G. S. Maddala and C. R. Rao, Amsterdam: North-Holland, pp. 241-268.

Elliott, G., Komunjer, I. and Timmermann, A. (2005), "Estimation and Testing of Forecast Rationality Under Flexible Loss", Review of Economic Studies 72(4): 1107-1125.

Elliott, G., Komunjer, I. and Timmermann, A. (2008), "Biases in Macroeconomic Forecasts: Irrationality or Asymmetric Loss", Journal of European Economic Association 6(1): 122157.

Elliott, G. and Timmermann, A. (2008), "Economic Forecasting", Journal of Economic Literature 46: $3-56$.

Granger, C. W. J. and Newbold, P. (1986), Forecasting Economic Time Series, 2nd ed., New York: Academic Press.

Komunjer, I. and Owyang M, T. (2012), "Multivariate Forecast Evaluation and Rationality Testing", The Review of Economics and Statistics 94(4): 1066-1080.

Mincer, J. and Zarnowitz, V. (1969), "The Evaluation of Economic Forecasts," in Economic Forecasts and Expectations, ed. J. Mincer, New York: National Bureau of Economic Research, pp. 81-111.

Newey, W. K. and West, K. D. (1987), "A Simple, Positive Semi-Definite, Heteroskedasticity and Autocorrelation Consistent Covariance Matrix", Econometrica 55: 703-708.

Patton, A. J. and Timmermann, A. (2007a), "Properties of Optimal Forecasts under Asymmetric Loss and Nonlinearity", Journal of Econometrics 140(2): 884-918.

Patton, A. J. and Timmermann, A. (2007b), "Testing Forecast Optimality under Unknown Loss", Journal of American Statistical Association 102(480): 1172-1184.

Patton, A. J. and Timmermann, A. (2010), "Why do Forecasters Disagree? Lessons from the Term Structure of Cross-Sectional Dispersion", Journal of Monetary Economics 57(7): 803-820.

Patton, A. J. and Timmermann, A. (2012), "Forecast Rationality Tests Based on Multi-Horizon Bounds", Journal of Business and Economic Statistics 30(1): 1-17.

Romer, C. D. and Romer, D. H. (2000), "Federal Reserve Information and the Behavior of Interest Rates", American Economic Review 90: 429-457.

Romer, C. D. and Romer, D. H. (2004), "Choosing the Federal Reserve Chair: Lessons from History", Journal of Economic Perspectives 18(1): 129-162. 
Rossi, B. (2012), "Comment on: Forecast Rationality Tests Based on Multi-Horizon Bounds", Journal of Business and Economic Statistics 30(1): 25-29.

Sims, C.A. (2002), "The Role of Models and Probabilities in the Monetary Policy Process", Brookings Papers on Economic Activity 2: 1-62.

Varian, H. R. (1975), "A Bayesian Approach to Real Estate Assessment", in Studies in Bayesian Econometrics and Statistics in Honor of Leonard J. Savage, eds. S. E. Fienberg and A. Zellner, Amsterdam: North-Holland, pp. 195-208. 
Table 1. The Full Sample Results of Estimates and Tests.

\begin{tabular}{|c|c|c|c|c|}
\hline \multirow[b]{2}{*}[\hat{f}_{t+h},y_{t+h}]{} & \multicolumn{2}{|c|}{ real output growth } & \multicolumn{2}{|c|}{ inflation } \\
\hline & $h=1$ & $h=4$ & $h=1$ & $h=4$ \\
\hline [Greenbook, Real-time] & & & & \\
\hline$\hat{\alpha}_{T, \tau}\left(\operatorname{se}\left(\hat{\alpha}_{T, \tau}\right)\right)$ & $0.572(.064)$ & $0.584(.075)$ & $0.530(.078)$ & $0.609(.095)$ \\
\hline$J_{T, \tau}(0.5)$ (p-value) & $1.700(.427)$ & $1.287(.526)$ & $0.148(.929)$ & $1.344(.511)$ \\
\hline$J_{T, \tau}\left(\hat{\alpha}_{T, \tau}\right)$ (p-value) & $0.429(.513)$ & $0.009(.925)$ & $0.001(.970)$ & $0.001(.971)$ \\
\hline [Greenbook, Revised] & & & & \\
\hline$\hat{\alpha}_{T, \tau}\left(\operatorname{se}\left(\hat{\alpha}_{T, \tau}\right)\right)$ & $0.438(.058)$ & $0.465(.072)$ & $0.454(.088)$ & $0.567(.109)$ \\
\hline$J_{T, \tau}(0.5)$ (p-value) & $1.249(.536)$ & $0.253(.880)$ & $0.445(.801)$ & $0.434(.805)$ \\
\hline$J_{T, \tau}\left(\hat{\alpha}_{T, \tau}\right)$ (p-value) & $0.056(.813)$ & $0.022(.882)$ & $0.171(.679)$ & $0.056(.813)$ \\
\hline [SPF, Real-time] & & & & \\
\hline$\hat{\alpha}_{T, \tau}\left(\operatorname{se}\left(\hat{\alpha}_{T, \tau}\right)\right)$ & $0.594(.059)$ & $0.651(.070)$ & $0.570(.067)$ & $0.749(.065)$ \\
\hline$J_{T, \tau}(0.5)$ (p-value) & $2.873(.238)$ & $5.200(.074)$ & $1.279(.528)$ & $14.009(.001)$ \\
\hline$J_{T, \tau}\left(\hat{\alpha}_{T, \tau}\right)$ (p-value) & $0.320(.572)$ & $0.568(.451)$ & $0.190(.663)$ & $0.483(.487)$ \\
\hline [SPF, Revised] & & & & \\
\hline$\hat{\alpha}_{T, \tau}\left(\operatorname{se}\left(\hat{\alpha}_{T, \tau}\right)\right)$ & $0.484(.059)$ & $0.568(.081)$ & $0.499(.082)$ & $0.699(.081)$ \\
\hline$J_{T, \tau}(0.5)(\mathrm{p}$-value $)$ & $0.367(.833)$ & $0.821(.663)$ & $0.729(.729)$ & $6.281(.043)$ \\
\hline$J_{T, \tau}\left(\hat{\alpha}_{T, \tau}\right)$ (p-value) & $0.292(.589)$ & $0.121(.728)$ & $0.632(.427)$ & $0.166(.684)$ \\
\hline
\end{tabular}

Notes: The results in this table are for the full sample period with $T=n$ and $\tau=1$. Reported in parentheses are the asymptotic standard error $s e\left(\hat{\alpha}_{T, \tau}\right)$ of $\hat{\alpha}_{T, \tau}$, and the p-values of the J statistics. $V_{t}=\left(\begin{array}{lll}1 & y_{t-1}\end{array}\right)^{\prime}$ is used. 
Figure 1. Output Growth Forecast $(h=1)$. Estimates and Tests with 1-step-ahead real output growth forecast: (a) and (b) with Greenbook and real-time data, (c) and (d) with Greenbook and revised data, (e) and (f) with SPF and real-time data, (g) and (h) with SPF and revised data. $V_{t}=\left(\begin{array}{ll}1 & y_{t-1}\end{array}\right)^{\prime}$ is used.

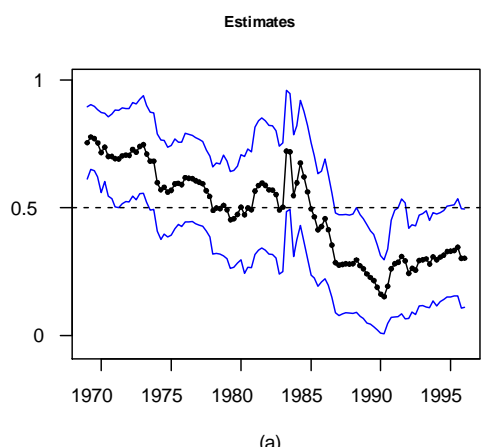

(a)
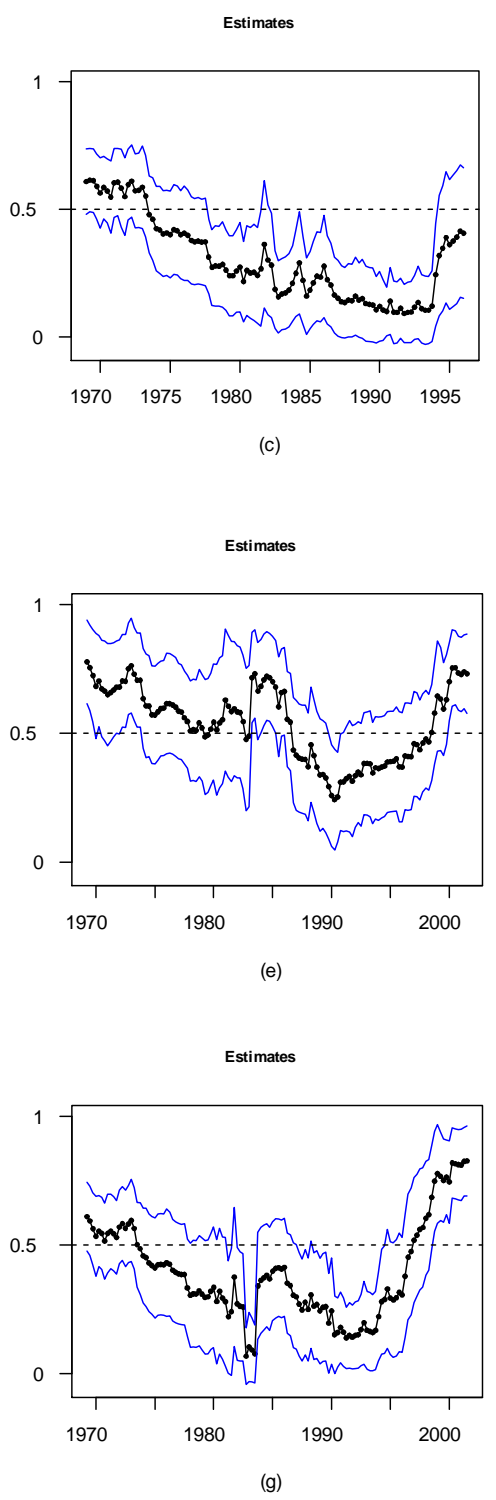
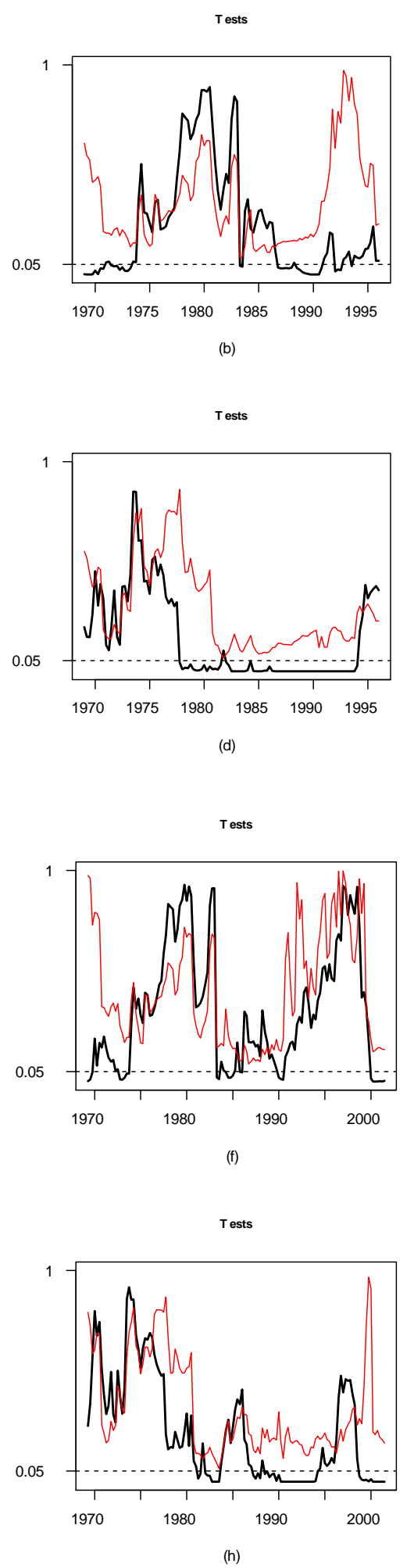
Figure 2. Output Growth Forecast $(h=4)$. Notes. Estimates and Tests with 4-step-ahead real output growth forecast: (a) and (b) with Greenbook and real-time data, (c) and (d) with Greenbook and revised data, (e) and (f) with SPF and real-time data, (g) and (h) with SPF and revised data. $V_{t}=\left(\begin{array}{ll}1 & y_{t-1}\end{array}\right)^{\prime}$ is used.
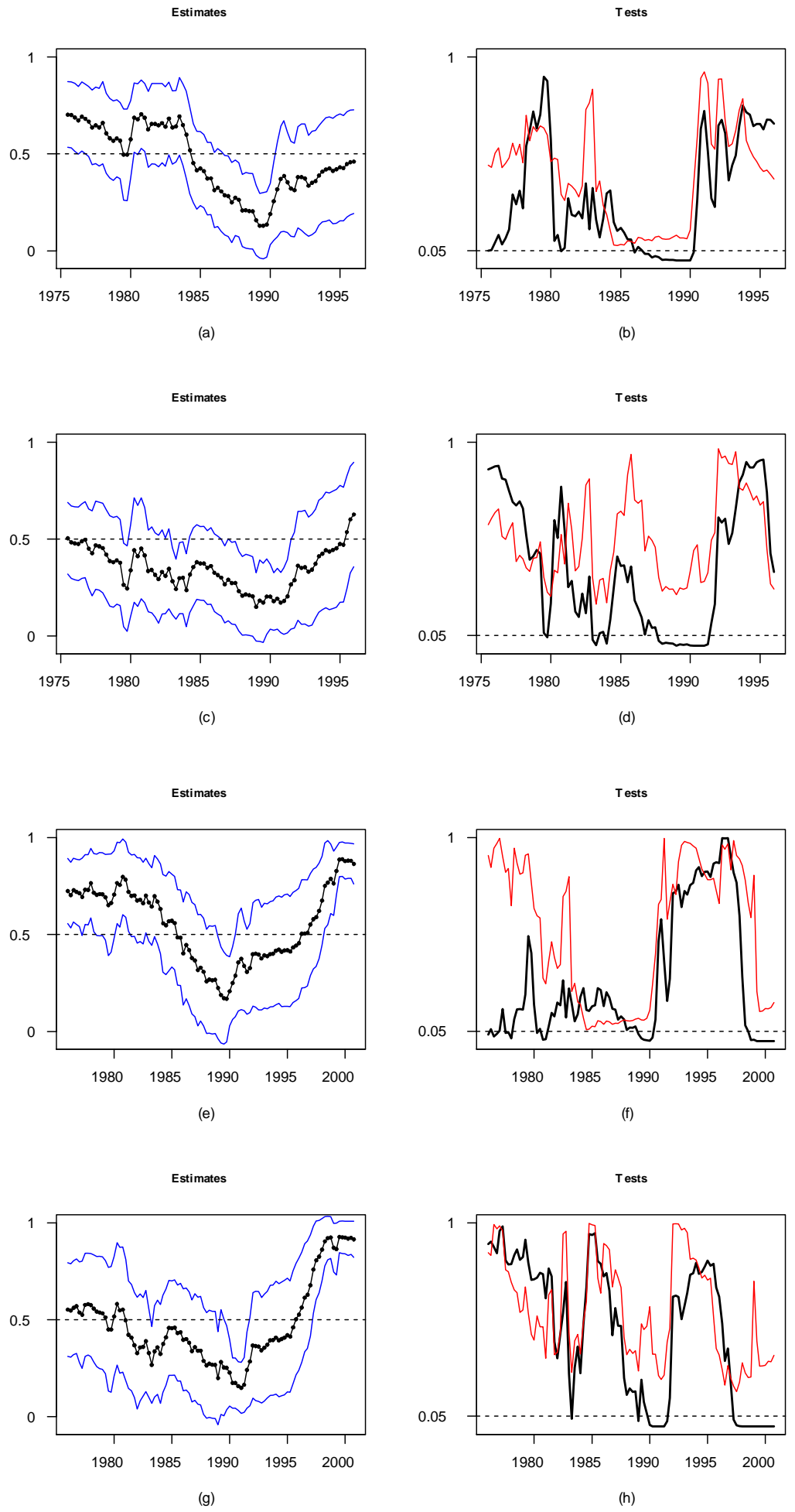
Figure 3. Inflation Forecast $(h=1)$. Notes. Estimates and Tests with 1-step-ahead inflation rate forecast: (a), (b) with Greenbook and real-time data; (c), (d) with Greenbook and revised data; (e), (f) with SPF and real-time data; (g), (h) with SPF and revised data. $V_{t}=\left(1 y_{t-1}\right)^{\prime}$.
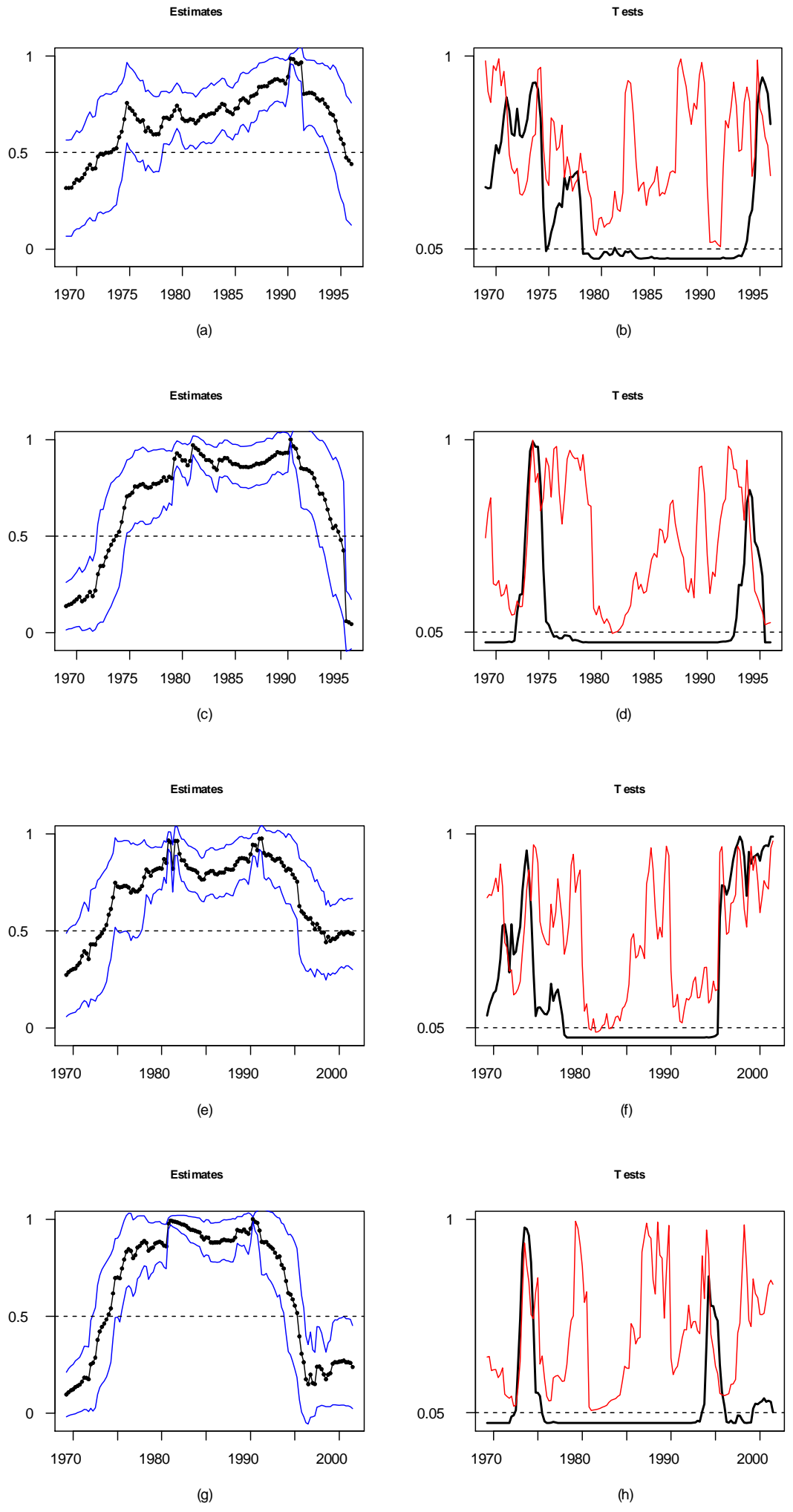
Figure 4. Inflation Forecast $(h=4)$. Notes. Estimates and Tests with 4-step-ahead inflation rate forecast: (a), (b) with Greenbook and real-time data; (c), (d) with Greenbook and revised data; (e), (f) with SPF and real-time data; (g), (h) with SPF and revised data. $V_{t}=\left(1 y_{t-1}\right)^{\prime}$.
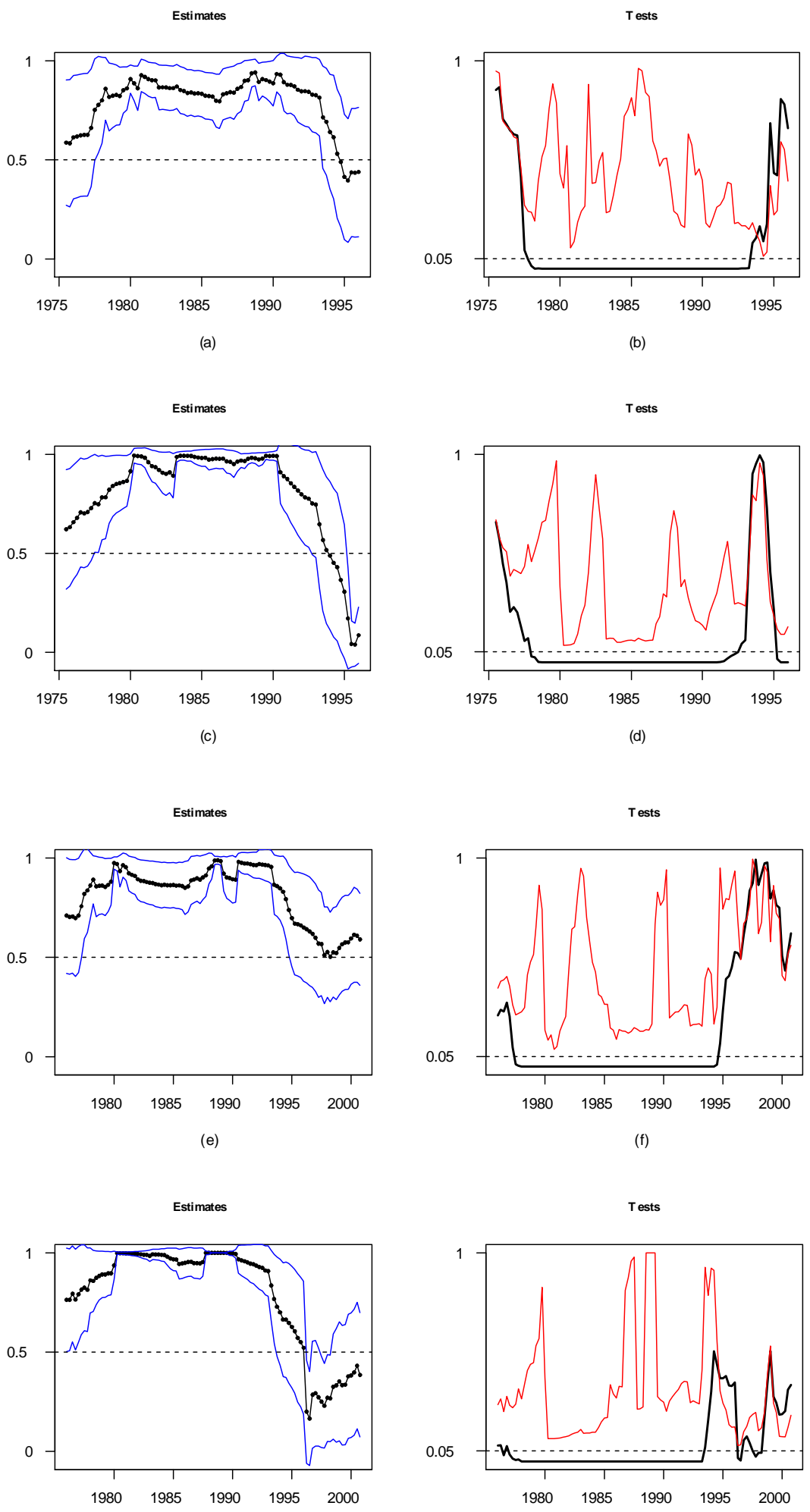

(g)

(h) 